\title{
Towards Human-Knee Orthosis Interaction Based on Adaptive Impedance Control Through Stiffness Adjustment*
}

\author{
Joana Figueiredo, Paulo Félix, Cristina P. Santos, and Juan C. Moreno, Member, IEEE
}

\begin{abstract}
Rehabilitation interventions involving powered, wearable lower limb orthoses that can provide high-challenging locomotor tasks for repetitive training sessions, mainly when assist-as-needed strategies, such as adaptive impedance control, are designed. In this study, the adaptive behavior was ensured by software control of the robotic stiffness involved in the human-knee orthosis interaction in function of the gait cycle and speed. To estimate the stiffness, we analyzed the interaction torque-angle characteristics with experimental data. The speedstiffness dependency was more evident when high stiffness values are demanded by the user's effort. Experimental evidence from five healthy subjects highlight that the adaptive control strategy provides a more comfortable, natural motion, and kinematic freedom as compared to the trajectory tracking control, allowing the user to contribute to the gait training. Future insights cover the implementation of gravitational compensation and real-time estimation and control of all inner dynamic properties of the impedance control law.
\end{abstract}

\section{INTRODUCTION}

Powered lower limb orthoses have been delivering a position-controlled robotic guidance, which does not resemble the manual assistance provided by a therapist that is usually compliant, and intuitively adaptive to the needs of the individual [1], [2]. Nevertheless, for a powered orthosis to be truly assistive, it must be capable of transit from passive to active behavior depending on the user's recovery stage [3]. In this sense, several adaptive control strategies, in particular, the assist-as-needed (AAN) control, have been investigated [2]. AAN training strategies can couple controllers capable of modulating the impedance of robots to promote a compliant human-robot interaction [4], [5].

The practical evidence of the gait rehabilitation based on AAN strategies has been highlighted through clinical studies using impedance control [6]. The first implementation of this controller in robotic devices for the gait rehabilitation was proposed in the Lokomat ${ }^{\circledR}$ (Hocoma, AG, US) [7]. Moreover, an impedance-controlled robot (LOPES: LOwer extremity Powered ExoSkeleton) was investigated during gait rehabilitation of spinal cord injury individuals [1]. However, these systems did not adapt the dynamic properties of the human-robot interaction, and they also limited the gait

\footnotetext{
* This work has been supported by the FCT - Fundação para a Ciência e Tecnologia - with the reference scholarship SFRH/BD/108309/2015, with the reference project UID/EEA/04436/2013, and by FEDER funds through the COMPETE 2020 - Programa Operacional Competitividade e Internacionalização (POCI) - with the reference project POCI-01-0145FEDER-006941
}

therapy to a clinical setting.

More recent studies have proposed adaptive impedance control [2], [6], and tailored the robot's dynamic properties (stiffness and/or damping) based on individuals' active joint torque estimated from the human-robot interaction force [2], [8]. The first work regarding the AAN robotic gait training using the adaptive impedance control scheme has been reported with Lokomat ${ }^{\circledR}$ [9]. Adaptive impedance control was also implemented on the powered orthosis developed by Hussain et al. [2], where the interaction torque was treated as an indication of the subject's effort. Similar approaches were introduced in [3], [10], although these studies did not validate the proposed strategy in the real environment.

Considering the potentialities of the AAN gait training, our research sought to implement and validate a hierarchical control architecture to tailor the impedance behavior of the human-orthosis interaction according to the user's effort. The proposed architecture gathers the control strategies presented in [2], [11] and introduces the stiffness's adjusting throughout four gait moments. We modulated the robotic stiffness of the human-orthosis interaction without introducing mechanical apparatus, such as spring. The stiffness's adjusting considered the subject-specific behavior of the gait assisted with the prescribed powered, wearable and untethered knee orthosis. According to authors' knowledge, no previous study has estimated the variation of the stiffness of the human-orthosis interaction throughout gait cycle for different gait phases.

The proposed control strategy also stands out from the other similar ones [2] by applying speed-dependent walking patterns in the adaptive impedance control law. This aspect makes the control architecture more versatile to assist the impaired locomotor function at different walking conditions. For comparison purposes, we also developed a predefined gait trajectory control to highlight the performance and the effects introduced on the gait assistance by the proposed architecture.

\section{METHODOLOGY}

\section{A. Powered Knee Orthotic System Design}

The powered knee orthosis involved in this study is intended for the gait rehabilitation of patients that present

Joana Figueiredo, Paulo Félix and Cristina P. Santos are now with the Center for MicroElectroMechanical Systems (CMEMS), University of Minho, Guimarães, Portugal (e-mail: id6003@alunos.uminho.pt, a61767@alunos.uminho.pt, cristina@dei.uminho.pt).

Juan C. Moreno is now with the Neural Rehabilitation Group, Cajal Institute, Spanish National Research Council, Spanish (e-mail jc.moreno@csic.es). 
deficits at the knee joint, such as genu recurvatum. This orthosis, depicted in Fig. 1, was designed to be worn by adults with height between 1.50 and $1.95 \mathrm{~m}$, and a maximum body weight of $100 \mathrm{~kg}$. During therapy, the orthosis's gait speed can be adjusted across different speeds from 0.5 to $1.8 \mathrm{~km} / \mathrm{h}$.

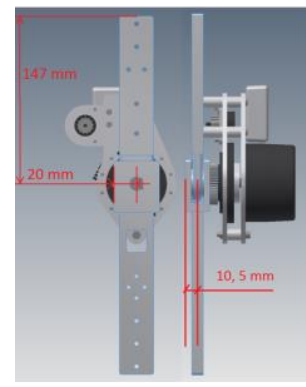

a)

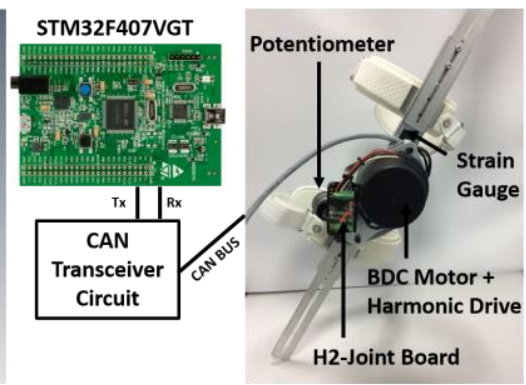

b)
Figure 1. Powered knee orthotic system: a) computer-aided design; b) real design, highlighting the communication between orthosis and control board.

The orthotic system is mainly composed by a powered knee orthosis, which consists of a modular 1-DOF joint (0$100^{\circ}$ range of motion - ROM) of the robotic lower-limb $\mathrm{H} 2$ exoskeleton (Technaid S.L., Spain), connected to the STM32F407VGT microcontroller through a Control Area Network (CAN), as shown in Fig. 1.b). This orthosis has embedded a precision potentiometer, strain gauges, and hall effect sensors (Fig. 1.b)) to monitor the joint's angle, userorthosis interaction torque, and the motor's angular speed, respectively. The actuation technology comprises an electrical actuator coupled to a strain wave gears to provide the required torque (high) and speed (low) for the application using brushless DC motor EC60-100W (Maxon) and gearbox CSD20-160-2A (Harmonic Drive). Moreover, we used an electronic board (H2-Joint Board) to manage the sensors acquisition, motor actuation, and communication (CAN) with the central microcontroller that runs the real-time control (STM32F407VG). The overall electronic system was powered by a LiFePO 4 battery of $24 \mathrm{~V}$ and $3 \mathrm{Ah}$. A detailed system's description is presented in [12].

Concerning the mechanical features, the powered orthotic system weights $2.02 \mathrm{~kg}$ with the power supply. The orthosis was attached to the lower limb by a 4-strap system, by placing the 2 upper straps on the thigh and the 2 lower straps on the shank. These straps can be adjusted, making the device suitable for patients with different anthropometric features. The minimal orthosis' length is $300 \mathrm{~mm}$ (Fig. 1.a)).

\section{B. Predefined Gait Trajectory Control}

For comparison purposes, we implemented a predefined gait trajectory control, in particular, the conventional position control through a closed-loop Proportional-IntegralDerivative (PID) controller, as illustrates Fig. 2. The knee sagittal plane physiological gait trajectories reported by Winter in [13] were used to define the reference joint angle trajectories. To improve the usability and flexibility of the controller, these reference trajectories were parameterized in accordance to the user-orthosis interaction's gait speed. As such, we applied (1) that describes the speed-dependent gait trajectory, found empirically, to set the frequency to update the reference trajectory, formed by 51 points as proposed by Winter, in function of gait speed.

$$
\text { Frequency }=-0.029 * \text { Gait Speed }+3.1
$$

The interaction between the exoskeleton and the user's limb should be as smooth as possible, avoiding abrupt movements that can cause discomfort to the user. For this reason, the position control should avoid oscillations in the trajectory and overshoot in each actuator's response. This behavior was achieved through the correct tuning of a PID controller by means Ziegler-Nichols method for the orthotic system. Concerning the practical application in a rehabilitation scenario, we used $K_{P}=90$ and $K_{I}=K_{D}=1.5$ as PID gains.

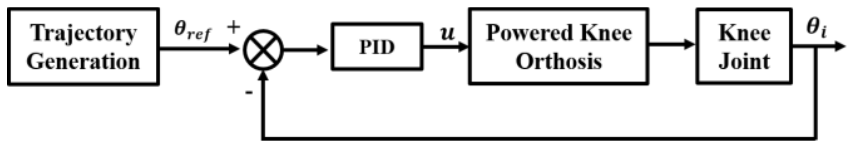

Figure 2. Diagram of the predefined gait trajectory control.

\section{Stiffness Estimation}

The modulation of stiffness involved in the walking, via activations of the agonistic and antagonistic muscles, is a relevant factor to ensure the compliance of lower extremity joints during locomotion [14]. In this sense, we estimated the robotic stiffness related to the human-orthosis interaction as the slope of the linear regression of the human-orthosis interaction torque at knee joint versus the knee angle. This approach is similar to the one explored by the concept of quasi-stiffness [14], [15].

The stiffness estimation took place in two phases. Firstly, we involved the healthy participants in walking trials at different gait speeds wearing the orthosis in passive mode (scenario where the torque reference is set to zero [12]). This was done to investigate how the stiffness associated with the human-orthosis interaction behaviors itself throughout the gait cycle and gait speed. Moreover, in this scenario there is no positive and negative contribution of the orthosis' actuation technology in the locomotion, only actuating the mechanical properties. In each trial, we monitored the humanorthosis interaction torque and the knee angle from the information recorded by the orthosis' embedded sensors. The second phase focused on the creation and analysis of the curve that relates the angle with the human-orthosis interaction torque at the knee joint. By applying the linear regression, we recognized, offline, the stiffness values and the gait moments in which they should be tailored in the impedance control law. The estimated stiffness values were normalized to $0-1$ range through the participant-specific percentage of the maximum stiffness due to operating limits of the actuation technology empirically observed for stiffness values higher than 1.5 N.m.

\section{Adaptive Impedance Control}

Based on the literature, proper functioning of an adaptive impedance controlled system implies the hierarchical organization of the control architecture [5], [16]. Thus, this study proposes the adaptive impedance control architecture illustrated in Fig. 3. This architecture is organized into three control levels. The generation of reference knee trajectory taken place on high-level control. Similarly to the position control, the reference knee trajectory $\left(\theta_{\text {ref }}\right)$ consisted on the knee angles found by Winter [13], which were updated in module "Trajectory Generation" through (1) (high-level control). In a future application, the "Trajectory Generation" 
module will involve key techniques to estimate the reference kinematic partner according to the user's locomotion intention.

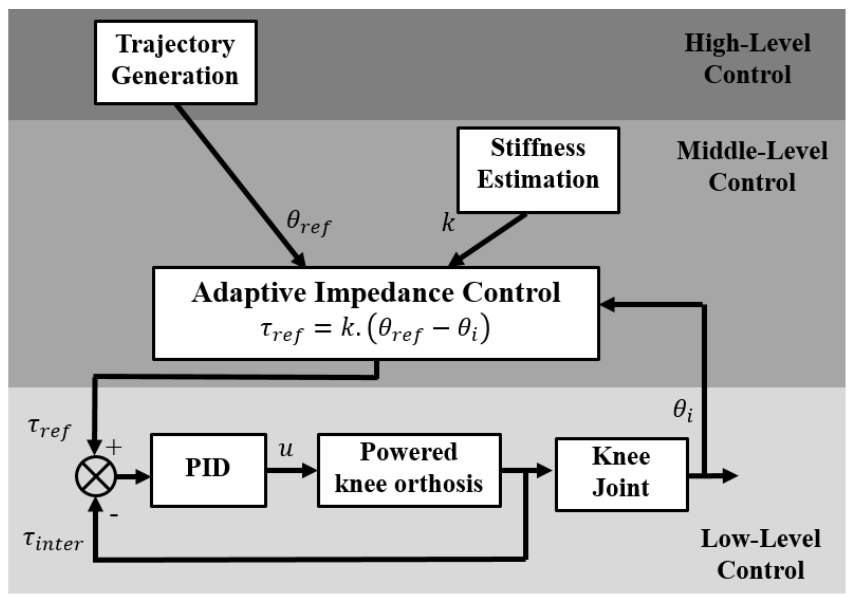

Figure 3. Hierarchical control architecture of the proposed adaptive impedance control.

The middle-level aims to estimate the reference humaninteraction torque $\left(\tau_{r e f}\right)$ by applying the impedance control law with adaptable stiffness values $(k)$ along gait cycle. The potentiometer measured the real knee joint angle $\left(\theta_{i}\right)$, assuming that the orthosis' joint was aligned with the subject's joint. The trajectory tracking errors from knee angles were continuously computed in real-time to calculate the knee joint torques $\left(\tau_{\text {ref }}\right)$ that guide the lower limbs on the reference trajectories. This determination was also affected by the stiffness $(k)$ estimated for the current gait moment through a finite state machine (FSM). Thus, the stiffness values were adjusted when the current gait moment match the gait phases implemented in the FSM (moments of the gait cycle where the stiffness should be updated). This procedure is performed by the "Stiffness Estimation" function integrated on the middle-level control. Due to mechanical constraints, we conducted a virtual adjusting of the stiffness instead of involving mechanical systems with variable stiffness behavior, as proposed by [3].

At the low-level control, we developed a PID control law in the torque form to guide the human-orthosis interaction torque $\left(\tau_{\text {inter }}{ }^{\prime}\right)$ in the direction of the desired torque by the user $\left(\tau_{\text {ref }}\right)$. The strain gauges provided the interaction torques ( $\left.\tau_{\text {inter }}\right)$ between the robotic orthosis and the human subject. These torque values describe the gravitational and inertial components, as well as the torques produced by the subject's joints, i.e., the torque generated by the human skeletal muscles, ligaments and other tissues surrounding joints. Based on the torque errors $\left(\tau_{\text {ref }}-\tau_{\text {inter }}\right)$, the PID controller sent a command to the actuation system to regulate the human-orthosis interaction considering the effort applied by the user. The control frequency was set to $1000 \mathrm{~Hz}$ at lowlevel and $100 \mathrm{~Hz}$ at middle-level control.

\section{E. Safety}

Different safety features were incorporated in the orthotic system. Due to mechanical issues, the joints ROM was reduced from 0 - 100 degrees to 3 - 98 degrees. As this interval is shorter than the human limits, the powered knee orthosis cannot damage the human legs by applying overextension or over flexion movements. Furthermore, the controller instabilities were avoided by adequate tune of the controller parameters' adjustments and by limiting the output PID commands. One last safety feature was concerning the proper and comfortable attachment and alignment of the orthosis with the lower limbs and knee joint, respectively.

\section{EXPERIMENTAL EVALUATION}

\section{A. Participants}

Five healthy neurologically intact subjects ( 2 females and 3 males) with no history of neurologic disorders gave informed consent and participated in the study. The main demographic characteristics of the involved subjects are $26.80 \pm 2.78$ years old, height of $1.68 \pm 0.07 \mathrm{~m}$, and weight of $64.60 \pm 8.5 \mathrm{~kg}$.

\section{B. Experimental Protocol}

First, the participants were asked to walk within the passive mode for $10 \mathrm{~min}$ on a treadmill so that they should become familiar with the robotic orthosis and training environment. Similar procedure was repeated for the robotic orthosis in assistive mode, either in predefined gait trajectory control and adaptive impedance control. In all situations, the orthosis was worn in the right limb, as shows Fig. 4.

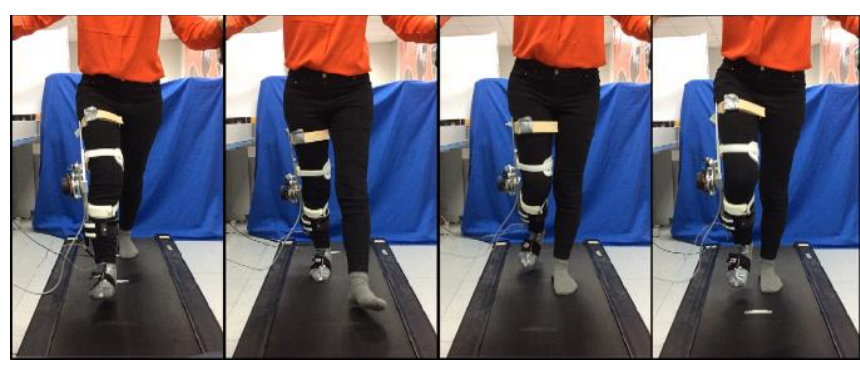

Figure 4. Frame sequence of an experiment conducted at $1.8 \mathrm{~km} / \mathrm{h}$ by a female subject wearing the powered knee orthosis in the passive mode.

To estimate the stiffness, the subjects performed 3 levelground walking trials of $2 \mathrm{~min}$ on a treadmill while wearing the powered knee orthosis in the passive mode. These trials were carried out at different gait speeds: 1, 1.3, 1.6 and 1.8 $\mathrm{km} / \mathrm{h}$. During these experiments, the subjects were asked to accomplish their normal gait patterns considering the imposed gait speed and orthotic system. A frame sequence of a female subject performing this protocol is illustrated in Fig. 4.

A similar protocol was carried out in the evaluation of the performance of both control strategies but lasting $12 \mathrm{~min}$. In this case, the participants walked $5 \mathrm{~min}$ in an assistive mode delivered by one control strategy (position or adaptive impedance control), resting $2 \mathrm{~min}$, and restarting the assisted walking with the other control strategy for $5 \mathrm{~min}$. The participants were randomly involved in both assistive modes (position and adaptive impedance control) in order to not influence their perception regarding the effects introduced by each control strategy. During these experiments, the subjects were encouraged to interact with the orthotic system, by following and opposing the reference trajectory.

We continuously monitored data from strain gauges and potentiometer. Additionally, in the end of protocol, each subject reported his/her perception, through a study-specific questionnaire, regarding both control strategies in terms of freedom of movement, providence of a natural motion, 
possibility or not to deviate from the reference knee trajectory, orthosis' ability to follow the human desired knee trajectory, and effort demanded from the user to perform the imposed trajectory. The patients also indicated their insights concerning the comfort and safety felt during the experiments.

\section{RESUlts AND DISCUSSION}

\section{A. Estimated Robotic Stiffness}

From the walking experiences with the knee powered orthosis in passive mode, we determined the robotic stiffness that modulates the interaction torque with the knee angle at different gait speeds. For space constraints, an instance of the dynamic relation of both parameters (torque and angle) is illustrated in Fig. 5 for 3 walking trials at $1.3 \mathrm{~km} / \mathrm{h}$. By applying the linear regression to each curve, we pointed out that the stiffness behaviors distinctly throughout the gait cycle. In particular, we verified that the stiffness changes its behavior in four particular gait moments: from heel-off $(\mathrm{HO})$ to toe-off (TO) event (HO->TO); from $\mathrm{TO}$ to middle mid-swing (MMSW) (TO-> MMSW); from MMSW to terminal swing phase (TS) (MMSW->TS); and, from TS to HO (TS->HO). Fig. 5 highlights this finding since these moments correspond to the ones when the linear regressions of the torque interaction vs knee angle curves present abrupt variations. Furthermore, from Fig. 5 we can outline that the stiffness values of HO->TO and MMSW->TS are similar (negative values) and higher than the ones required in TO->MMSW and TS->HO (positive values), which are also closer.

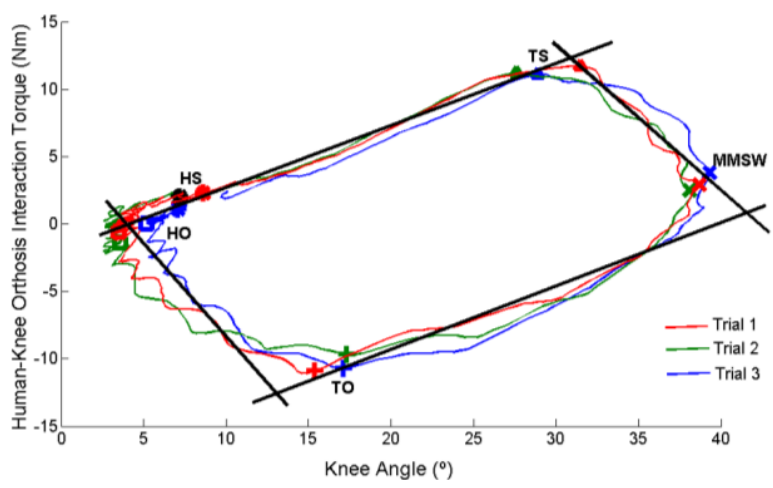

Figure 5. Human-knee orthosis interaction torque $v s$ knee angle of 3 walking trials of a male subject walking at $1.3 \mathrm{~km} / \mathrm{h}$ on the treadmill.

We determined the stiffness values for the four gait moments from the slope of the linear regression of torque interaction $v s$ knee angle curve. Table 1 details the module of mean and standard deviation (std) of the estimated stiffness, in function of the user' weight, for each considered gait speed and identified gait moment. The normalized values (ranging 0 - 1) in function of the maximum stiffness are also outlined.

The module of the estimated stiffness values was plotted (see Fig. 6) to facilitate the interpretation of their variation with the gait cycle and speed. Fig. 6 highlights the similarity of stiffness values in HO-> TO and MMSW->TS, and in TO$>$ MMSW and TS->HO, as observed in Fig. 5. This finding indicates that the human-orthosis interaction should be more stiff to perform the TO event and change the limb progression's direction, i.e., from the knee flexion to knee extension. Therefore, a higher impedance (ranging from 0.012 \pm 0.003 to $0.039 \pm 0.004 \mathrm{Nm} /{ }^{\circ} \mathrm{kg}$, Table 1) is required to prepare the assisted limb for the stance-swing transition and to accelerate it in the advancing of the opposite stance limb since these gait motions require a high interaction torque for a low excursion. On the other hand, by analyzing Fig. 5 and Fig. 6 , we found that from the initial to middle-swing phase, and from the terminal to middle-stance phase the human-orthosis interaction should be more compliant. In fact, the beginning of stance and swing phases demands a lower interaction stiffness $\left(0.007 \pm 0.005 \mathrm{Nm} /{ }^{\circ} \mathrm{kg}\right.$, Table 1$)$ to achieve a high knee ROM.

TABLE I. ESTIMATED STIFFNESS VALUES

\begin{tabular}{|c|c|c|c|c|}
\hline \multirow{2}{*}{$\begin{array}{c}\text { Gait } \\
\text { speed } \\
(\mathbf{k m} / \mathbf{h})\end{array}$} & \multirow{2}{*}{$\begin{array}{c}\text { Gait } \\
\text { Moments }\end{array}$} & \multicolumn{2}{|c|}{ Stiffness (N.m/ $\left.{ }^{\circ} \mathbf{k g}\right)$} & \multirow{2}{*}{$\begin{array}{c}\text { Stiffness } \\
\text { Normalized } \\
\left(\text { N.m/ }{ }^{\circ} \mathbf{k g}\right)\end{array}$} \\
\hline & & Mean & Std & \\
\hline \multirow{4}{*}{1} & $\mathrm{HO}->\mathrm{TO}$ & 0.012 & 0.003 & 1.0 \\
\hline & $\begin{array}{c}\text { TO -> } \\
\text { MMSW }\end{array}$ & 0.0085 & 0.001 & 0.71 \\
\hline & $\begin{array}{c}\text { MMSW-> } \\
\text { TS }\end{array}$ & 0.012 & 0.003 & 1.0 \\
\hline & $\mathrm{TS}->\mathrm{HO}$ & 0.008 & 0.002 & 0.71 \\
\hline \multirow{4}{*}{1.3} & $\mathrm{HO}->\mathrm{TO}$ & 0.020 & 0.005 & 0.95 \\
\hline & $\begin{array}{c}\text { TO -> } \\
\text { MMSW }\end{array}$ & 0.008 & 0.001 & 0.38 \\
\hline & $\begin{array}{c}\text { MMSW-> } \\
\text { TS } \\
\end{array}$ & 0.021 & 0.004 & 1.0 \\
\hline & $\mathrm{TS}->\mathrm{HO}$ & 0.008 & 0.003 & 0.38 \\
\hline \multirow{4}{*}{1.6} & $\mathrm{HO}->\mathrm{TO}$ & 0.024 & 0.004 & 1.0 \\
\hline & $\begin{array}{c}\text { TO -> } \\
\text { MMSW }\end{array}$ & 0.008 & 0.001 & 0.33 \\
\hline & $\begin{array}{c}\text { MMSW-> } \\
\text { TS }\end{array}$ & 0.023 & 0.004 & 0.96 \\
\hline & $\mathrm{TS}->\mathrm{HO}$ & 0.007 & 0.0005 & 0.29 \\
\hline \multirow{4}{*}{1.8} & $\mathrm{HO}->\mathrm{TO}$ & 0.036 & 0.002 & 0.92 \\
\hline & $\begin{array}{c}\text { TO -> } \\
\text { MMSW }\end{array}$ & 0.007 & 0.003 & 0.18 \\
\hline & $\begin{array}{c}\text { MMSW-> } \\
\text { TS }\end{array}$ & 0.039 & 0.004 & 1.0 \\
\hline & $\mathrm{TS}->\mathrm{HO}$ & 0.009 & 0.002 & 0.23 \\
\hline
\end{tabular}

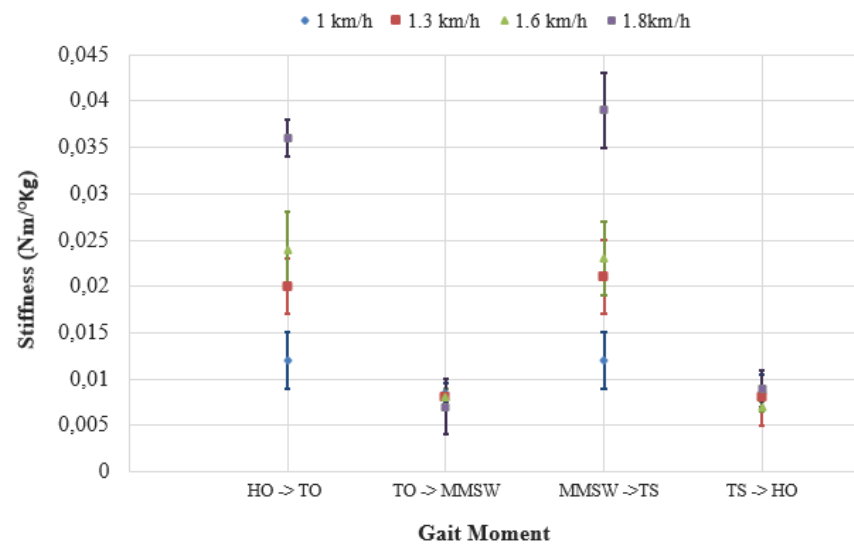

Figure 6. Mean and std values of the estimated stiffness throughout gait cycle for different gait speeds.

The results presented in Table 1 and Fig. 6 also show that the stiffness of the human-orthosis interaction varies positively with the gait speed, i.e., as higher is the gait speed higher is the stiffness. This relation is more pronouncing in transitions HO$>$ TO and MMSW->TS, i.e., the stiffness's dependency on speed is more evident in scenarios that involve high stiffness values. 
Based on knowledge of the authors, there is no study in the literature that has empirically estimated the stiffness that modulates the human-orthosis interaction. Consequently, the stiffness values estimated in this work diverge from the ones found in the literature for the orthotic-gait training [17], [18]. In fact, the studies disclosed in the literature focus on the investigation of the stiffness of the healthy human joint, instead explore the stiffness of the human-orthosis interface, to define how the mechanical systems (e.g. springs) attached to the orthosis have to emulate the dynamic properties of a healthy joint [14], [19], [20]. Thus, we cannot compare the outcomes obtained with well-established stiffness values mainly because the estimated values depend on the dynamic behavior of the prescribed orthotic system. Nevertheless, the findings disclosed in this study are according with the ones presented in the literature regarding the variation of stiffness with the gait speed (positively correlated) [14], [17], [18]. Additionally, the estimated stiffness values are within the same order of magnitude of the values found in [19], [17], [18].

\section{B. Adaptive Impedance Control vs Trajectory Control}

During the walking experiments in the assistive mode, the reference and real values of the knee trajectories and humanknee orthosis interaction were monitored to investigate and compare the performance of both implemented control strategies. By analyzing Fig. 7, we verified that the real knee trajectory is not affected by the human-orthosis interaction torque independently of its magnitude and contribution (positive and negative). In particular, at $13 \mathrm{~s}$ of walking trials at $1 \mathrm{~km} / \mathrm{h}$ (Fig. 7.), the participant augmented the interaction with the wearable orthosis (increased torque interaction) but no effects were observed in the real knee trajectory. This finding indicates that the trajectory control is not suitable for AAN gait training since it imposes to the joint a predefined trajectory without considering the human participation. Such observation is in accordance with the position control's outcomes reported in the literature [1], [2], [6].

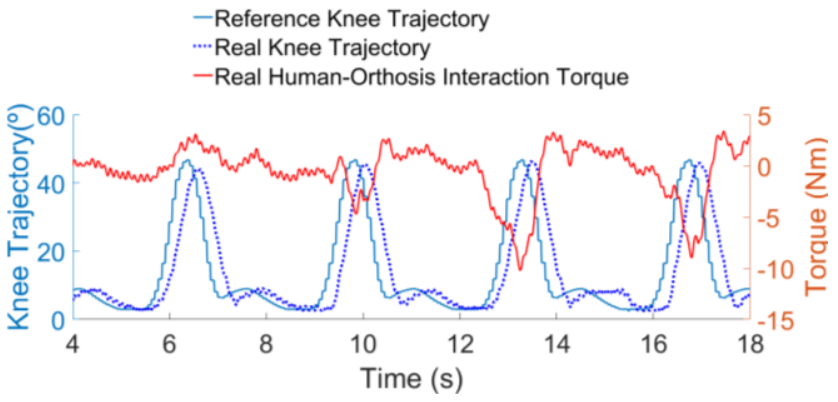

Figure 7. Outcomes of the walking experiments at $1 \mathrm{~km} / \mathrm{h}$ assisted with position control.

On the other hand, the outcomes obtained in the adaptive impedance control, illustrated in Fig. 8, show that the increasing the torque interaction leads to an augment of the real knee trajectory, which may exceed the ROM of the reference trajectory. Nevertheless, we verified that the real knee angle never exceeds the imposed limits for orthosis' ROM due to the suitable application of the safety features. Fig. 8 also outlines that when a participant interacts more with the orthosis (e.g. from 86 to $91 \mathrm{~s}$ of the walking trial at $1 \mathrm{~km} / \mathrm{h}$ ), a lower reference interaction torque is determined by the impedance control law since the real trajectory is more aligned with the reference one, mainly at the beginning of each walking cycle. Subsequently, the trajectory tracking errors decrease, resulting in a proportional reduction (dependent on the stiffness value) of the reference interaction torque. These findings support the suitable application of the adaptive impedance control as an AAN strategy since the proposed strategy guides the lower limbs by considering simultaneously the user's effort (from the human-orthosis interaction torque) and predefined gait patterns [2], [6], [11]. Additionally, in opposition to trajectory control, this strategy considers the user's intended motion since as higher is torque interaction higher is the ROM of the real joint.

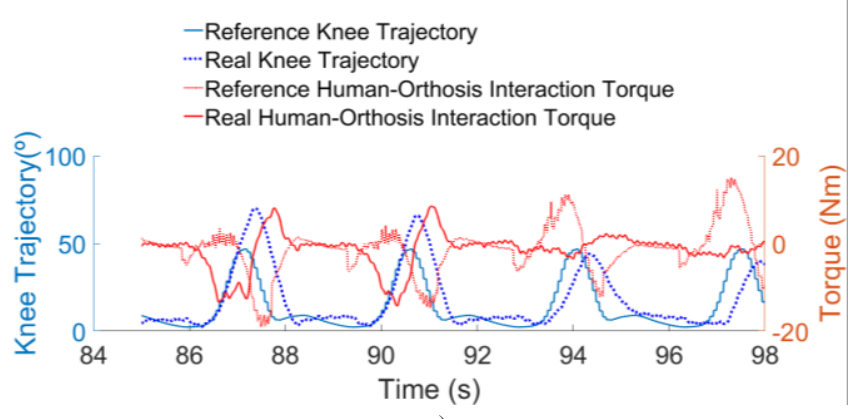

a)

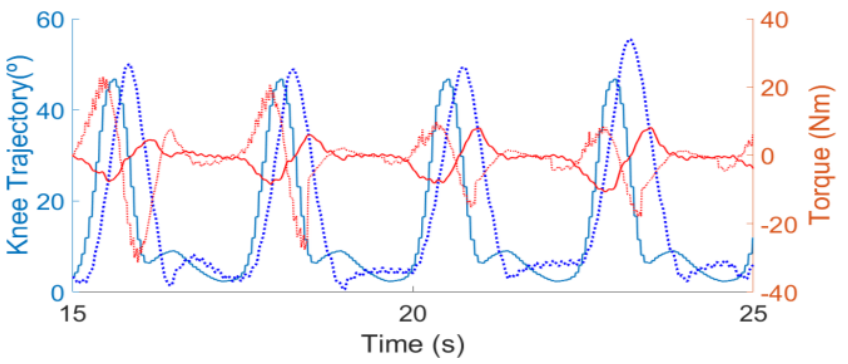

b)

Figure 8. Outcomes of the walking experiments assisted with adaptive impedance control: a) $1 \mathrm{~km} / \mathrm{h}$; b) $1.6 \mathrm{~km} / \mathrm{h}$.

Concerning the delay of the control mechanism, Fig.7 and Fig. 8 point out that there is a delay in the real knee trajectory comparing to the one imposed due to the delayed response time exhibited by the PID controller. A maximal delay of 0.25 $\mathrm{s}$ was observed in position control. However, in the adaptive strategy, as higher is the torque interaction, the higher is the synchronization of the real knee trajectory with the expected one, decreasing the delay of the knee trajectory.

For the comparison of the adaptive impedance control with the trajectory control relating clinical relevance and usability, we consider the secondary outcomes reported by the participants. In all cases, the subjects felt differences between both control strategies when wore the orthotic system properly attached and aligned with the knee joint. In fact, they reported that the adaptive impedance control delivers a more comfortable and natural motion, and possibilities to the user deviate himself from the imposed robotic trajectory, as indicated in Fig. 8. The increasing freedom of movement was more pronounced when low stiffness values were involved in the impedance control law. Additionally, the subjects straightforwardly perceived that the impedance controller allows more interaction since when they tried to deviate from the trajectory imposed by the orthosis in position control, they 
felt the orthosis more rigid, as it is an extra weight comparatively to the one perceived in the impedance control.

One last aspect reported by the users is related to the stiffness's variation. For low stiffness values (more compliant behavior), the subjects had to apply more effort to perform the same trajectory imposed in trials that used high stiffness values (more stiff behavior). Such observation is according to [2]. Based on the user's perception, we also outlined that the subject-specific stiffness's adjusting implemented in this study responds to the patient's needs since the stiff behavior was activated in the gait moments that demand more effort.

During the adaptive impedance control mode, the maximum angular deviations from the desired knee joint angle trajectories were below $20^{\circ}$. This flexibility suggests that the proposed adaptive control creates a "virtual tunnel" along the reference trajectory of the patient's joints to state the amount of supportive force the subject deviates from the desired motion. A similar approach was disclosed in [8].

\section{B. Limitations and Future Perspectives}

The performance of an impedance control is determined by the precision of the position and torque sensors. For this purpose, we constantly checked the calibration of both sensory systems during the experiments. The actuator's torque precision and bandwidth also influence the performance of the impedance control, mainly when the actuation system augments the total inertia apparent. To minimize this negative influence, in future contributions, we should integrate into low-level control a torque compensation feed-forwarded to compensate the gravity. Another important future research question concerns in the real-time adjustment of the reference trajectory according to the patient's effort. Future perspectives also include the estimation and control of other inner properties (damping and inertia) of the impedance control. A last future challenge covers the improvement of the PID parameters' tuning to overcome the overshoot and the delay in the trajectory.

\section{CONCLUSIONS}

This study conducted an innovative investigation regarding the robotic stiffness that modulates the humanorthotic system interaction. The outcomes highlight the importance of the stiffness's adjusting in function of the gait cycle and gait speed. The primary and secondary experimental outcomes demonstrate that the adaptive control strategy based on virtual stiffness's modulation is able to recognize and tailor the therapy according to user's effort. Compared to position-controlled robotic training, the adaptive control strategy delivers a more comfortable and natural motion. All subjects also reported more kinematic freedom as compared to the tracking mode as they have the liberty to actively contribute in the gait training process. Experimental evidences pointed out that the proposed impedance control creates a "virtual tunnel" around the reference gait pattern, being its magnitude dependent on the stiffness value. Thus, the first advances introduced in this work towards an adaptive robotic therapy have shown to be suitable for task-specific and intensive training sessions.

\section{REFERENCES}

[1] B. M. Fleerkotte, B. Koopman, J. H. Buurke, E. H. F. van Asseldonk, H. van der Kooij, and J. S. Rietman, "The effect of impedancecontrolled robotic gait training on walking ability and quality in individuals with chronic incomplete spinal cord injury: an explorative study.," J. Neuroeng. Rehabil., vol. 11, p. 26, Jan. 2014.

[2] S. Hussain, S. Q. Xie, S. Member, and P. K. Jamwal, "Adaptive Impedance Control of a Robotic Orthosis for Gait Rehabilitation," vol. 43, no. 3, pp. 1025-1034, 2013.

[3] G. Aguirre-Ollinger, J. E. Colgate, M. A. Peshkin, and A. Goswami, "Active-impedance control of a lower-limb assistive exoskeleton," 2007 IEEE 10th Int. Conf. Rehabil. Robot. ICORR'07, vol. 0, no. c, pp. 188-195, 2007.

[4] W. Meng, Q. Liu, Z. Zhou, Q. Ai, B. Sheng, and S. Xie, "Recent development of mechanisms and control strategies for robot-assisted lower limb rehabilitation," Mechatronics, vol. 31, pp. 132-145, 2015.

[5] J. C. P. Ibarra and A. A. G. Siqueira, "Impedance control of rehabilitation robots for lower limbs. Review," Proc. - 2nd SBR Brazilian Robot. Symp. 11th LARS Lat. Am. Robot. Symp. 6th Rob. Work. Appl. Robot. Autom. SBR LARS Rob. 2014 - Part Jt. Conf. Robot. Intell. Syst. , pp. 235-240, 2015.

[6] J. Cao, S. Q. Xie, R. Das, and G. L. Zhu, "Control strategies for effective robot assisted gait rehabilitation: the state of art and future prospects.," Med. Eng. Phys., vol. 36, no. 12, pp. 1555-66, 2014

[7] S. Jezernik, G. Colombo, and M. Morari, "Automatic gait-pattern adaptation algorithms for rehabilitation with a 4-DOF robotic orthosis," IEEE Trans. Robot. Autom., vol. 20, no. 3, pp. 574-582, 2004.

[8] M. Bortole, A. Venkatakrishnan, F. Zhu, J. C. Moreno, G. E. Francisco, J. L. Pons, and J. L. Contreras-Vidal, "The H2 robotic exoskeleton for gait rehabilitation after stroke: early findings from a clinical study," $J$. Neuroeng. Rehabil., vol. 12, no. 1, p. 54, 2015.

[9] R. Riener, L. Lünenburger, S. Jezernik, M. Anderschitz, G. Colombo, and V. Dietz, "Patient-cooperative strategies for robot-aided treadmill training: First experimental results," IEEE Trans. Neural Syst. Rehabil. Eng., vol. 13, no. 3, pp. 380-394, 2005.

[10] W. M. Dos Santos and A. A. G. Siqueira, "Optimal impedance control for robot-Aided rehabilitation of walking based on estimation of patient behavior," Proc. IEEE RAS EMBS Int. Conf. Biomed. Robot. Biomechatronics, vol. 2016-July, pp. 1023-1028, 2016.

[11] G. Aguirre-Ollinger, U. Nagarajan, and A. Goswami, "An admittance shaping controller for exoskeleton assistance of the lower extremities," Auton. Robots, vol. 40, no. 4, pp. 701-728, 2016.

[12] P. Felix, J. Figueiredo, C. P. Santos, and J. C. Moreno, "Electronic Design and Validation of Powered Knee Orthosis System with Werable Sensors," 17th Int. Conf. Auton. Robot Syst. Compet, 2017.

[13] D. Winter, Biomechanics and Motor Control of Human Movement, 4 th., vol. 2nd. 2009.

[14] G. M. Gu, S. Kyeong, D. S. Park, and J. Kim, "SMAFO: Stiffness modulated Ankle Foot Orthosis for a patient with foot drop," IEEE Int. Conf. Rehabil. Robot., vol. 2015-Septe, pp. 543-548, 2015.

[15] K. Shamaei, G. S. Sawicki, and A. M. Dollar, "Estimation of QuasiStiffness and Propulsive Work of the Human Ankle in the Stance Phase of Walking," PLoS One, vol. 8, no. 3, 2013.

[16] M. R. Tucker, J. Olivier, A. Pagel, H. Bleuler, M. Bouri, O. Lambercy, J. D. R. Millan, R. Riener, H. Vallery, and R. Gassert, "Control Strategies for Active Lower Extremity Prosthetics and Orthotics: A Review.," J. Neuroeng. Rehabil., vol. 12, no. 1, p. 1, Jan. 2015.

[17] K. Shamaei, G. S. Sawicki, and A. M. Dollar, "Estimation of QuasiStiffness of the Human Knee in the Stance Phase of Walking," PLoS One, vol. 8, no. 12, 2013.

[18] S. Pfeifer, R. Riener, and H. Vallery, "Knee stiffness estimation in physiological gait," Conf. Proc. ... Annu. Int. Conf. IEEE Eng. Med. Biol. Soc. IEEE Eng. Med. Biol. Soc. Annu. Conf., vol. 2014, no. October 2016, pp. 1607-1610, 2014.

[19] K. Shamaei, M. Cenciarini, A. A. Adams, K. N. Gregorczyk, J. M. Schiffman, and A. M. Dollar, "Design and evaluation of a quasi-passive knee exoskeleton for investigation of motor adaptation in lower extremity joints," IEEE Trans. Biomed. Eng., vol. 61, no. 6, pp. 1809 1821, 2014.

[20] Y. L. Kerkum, A. I. Buizer, J. C. Van Den Noort, J. G. Becher, J. Harlaar, and M. A. Brehm, "The effects of varying ankle foot orthosis stiffness on gait in children with spastic cerebral palsy who walk with excessive knee flexion," PLoS One, vol. 10, no. 11, 2015. 
